\title{
QUEEN'S
UNIVERSITY
BELFAST
}

\section{Genetic renal disorders}

Maxwell, A. (2019). Genetic renal disorders. Medicine. https://doi.org/10.1016/j.mpmed.2019.05.007

\section{Published in: \\ Medicine}

\section{Document Version:}

Peer reviewed version

Queen's University Belfast - Research Portal:

Link to publication record in Queen's University Belfast Research Portal

\section{Publisher rights}

(C) 2019 Elsevier Ltd.

This manuscript version is made available under the CC-BY-NC-ND 4.0 license http://creativecommons.org/licenses/by-nc-nd/4.0/,which permits distribution and reproduction for non-commercial purposes, provided the author and source are cited

\section{General rights}

Copyright for the publications made accessible via the Queen's University Belfast Research Portal is retained by the author(s) and / or other copyright owners and it is a condition of accessing these publications that users recognise and abide by the legal requirements associated with these rights.

\section{Take down policy}

The Research Portal is Queen's institutional repository that provides access to Queen's research output. Every effort has been made to ensure that content in the Research Portal does not infringe any person's rights, or applicable UK laws. If you discover content in the Research Portal that you believe breaches copyright or violates any law, please contact openaccess@qub.ac.uk. 


\title{
Genetic renal disorders
}

\author{
Peter Maxwell
}

Peter Maxwell MD PhD FRCP is Consultant Nephrologist at Belfast City Hospital and Professor of Renal Medicine at Queen's University Belfast, UK. Competing interests: none declared.

\begin{abstract}
Genetic disorders of renal structure and function are recognized causes of end-stage renal disease (ESRD) requiring renal replacement therapy. A family history of haematuria, urinary tract infection or ESRD can prompt the clinician to consider an underlying renal genetic disorder. In practice, the most common inherited renal condition is autosomal dominant polycystic kidney disease (ADPKD), characterized by multiple kidney cysts associated with hypertension and later progression to chronic kidney disease (CKD). Insights into the cell biology of ADPKD have resulted in new therapy to limit cyst growth and slow the progression of CKD. Non-visible haematuria can present a diagnostic challenge because it has so many possible causes. Mutations in genes encoding collagen proteins within the glomerular basement membrane can disrupt its normal barrier function. Thin basement membrane nephropathy, caused by glomerular basement membrane collagen gene mutations, can cause familial haematuria that normally has a good long-term prognosis. Alport's syndrome is a rarer and genetically heterogeneous condition classically leading to CKD associated with sensorineural deafness in men inheriting an X-linked gene defect. Single-gene defects can also cause diverse renal tubular disorders, such as predisposition to hypertension with associated electrolyte imbalance, renal calculi, renal tubular acidosis or diabetes insipidus. Gene mutations responsible for familial renal cancer syndromes, such as tuberous sclerosis complex, Birt-Hogg-Dubé syndrome and von Hippel-Lindau disease, are rare but important to recognize so that long-term surveillance for cancers is commenced.
\end{abstract}

\section{Keywords}

Alport's syndrome; non-visible haematuria; polycystic kidneys; renal genetics; thin basement membrane nephropathy

\section{Key points}

-Autosomal dominant polycystic kidney disease (ADPKD) is the most common renal genetic disorder in general practice and is disproportionately common in undergraduate and postgraduate examinations -Insights from cell biology have been translated into novel therapies for ADPKD

-NICE has approved tolvaptan, a vasopressin receptor antagonist, for clinical use to slow progression of cyst development and renal failure in adults with ADPKD

-Asymptomatic non-visible haematuria is common, and investigation of younger adults may identify mutations in genes encoding proteins that form part of the glomerular basement membrane

\section{Introduction}

Up to $10 \%$ of new patients commencing renal replacement therapy (RRT) annually in the UK have renal genetic disorders. ${ }^{1}$ Together these represent an important cause of end-stage renal disease (ESRD) after diabetes mellitus, hypertension, glomerulonephritis or pyelonephritis. ${ }^{1}$ The common presentations of kidney disease - proteinuria, haematuria, electrolyte disorders and anatomical kidney abnormalities - can have an underlying genetic cause. Some monogenic renal disorders are relatively common; for example, a UK general practitioner can provide care for several individuals with polycystic kidney disease. Rarer inherited disorders can be responsible for the development of non-visible (microscopic) haematuria, nephrolithiasis 
and renal tubular disorders that cause electrolyte imbalance (Table 1). The possibility of an inherited renal defect should be considered in patients presenting with these findings.

\section{Background}

Common causes of kidney failure such as diabetic nephropathy, glomerulonephritis and hypertension have complex aetiologies resulting from dynamic interactions between environmental factors and an individual's genetic susceptibility to kidney disease. Kidney disease occurring as a direct consequence of an inherited gene mutation, such as autosomal dominant polycystic kidney disease (ADPKD), can still be modified by additional environmental and genetic factors.

Finding a gene mutation associated with a particular renal disorder is the initial step in understanding how DNA sequence variants are related to changes in the amount or function of translated proteins. A monogenic disorder, such as ADPKD, which has easily recognized clinical features (phenotype), can arise from different mutations (genotypes) - unique for individual families (private mutations) - in either of two distinct genes (PKD1, PKD2). This has practical consequences for genetic counselling within an affected family as it can be technically challenging to identify the causative pathogenic mutation. ADPKD mutations can lead to early truncation of the transcribed protein (because of a premature stop codon) or provide more subtle alteration of the protein's function by altering its amino acid sequence.

\section{Identifying renal genetic disorders}

Obtaining a family history can help to identify a genetic kidney disease, and constructing an extended family pedigree can provide evidence of a Mendelian inheritance pattern. Monogenic renal disorders can be inherited in an autosomal dominant, autosomal recessive, X-linked dominant or X-linked recessive fashion. ${ }^{2}$ Recessive disorders typically present in childhood, whereas dominant disorders are more usually identified in adulthood when a 'second-hit' somatic mutation of the normal inherited allele occurs (e.g. von HippelLindau disease). For X-linked diseases (e.g. Alport's syndrome), male individuals are more severely affected at an earlier age than females. Female patients have a varying severity of $X$-linked renal disease, reflecting the degree of lyonization (the random inactivation of maternal versus paternal $X$ chromosomes in cells). In addition, various genetic and environmental modifiers can alter the age at presentation of a monogenic renal disorder: the age at which ESRD occurs in ADPKD can vary widely within a family despite the affected members inheriting an identical PKD1 mutation.

Renal malformations (around 1:200 pregnancies) are associated with altered kidney development and differentiation. The pathogenesis of these congenital structural abnormalities of the kidney and urinary tracts (CAKUT) is complex and likely to be caused by combinations of genetic, epigenetic and environmental factors (teratogens). Approximately $50 \%$ of children with ESRD have CAKUT; almost half of these are associated with lower urinary tract obstruction resulting from posterior urethral valves in boys. ${ }^{1,2}$

Almost $30 \%$ of children who present with urinary tract infection have vesico-ureteric reflux (VUR). It is worthwhile establishing the family history of children with both urinary tract infection and VUR because VUR is reported to be present in $20-40 \%$ of the siblings and offspring of affected children. There is a genetic susceptibility to VUR, but no consistently causative genetic loci have been identified. This reflects the phenomenon of genetic heterogeneity in which different genes in each family are responsible for the VUR phenotype. Close working relationships between general practitioners, paediatricians, nephrologists and geneticists are necessary for efficient clinical assessment of affected children with CAKUT and VUR, and to ensure accurate genetic counselling for families.

\section{Polycystic kidney disease}

\section{Genetics}

ADPKD is the most common inherited renal disorder, with a reported prevalence of 1:500 to 1:2000 (Table

2). ADPKD is caused by germline mutations in two genes: PKD1 (chromosome 16p13.3-p13.1) and PKD2 (chromosome 4q21-q23). Mutations in PKD1 and PKD2 are responsible for $85 \%$ and $15 \%$ of ADPKD cases, 
respectively. ${ }^{3}$ Over 1700 unique mutations affecting these two genes have been identified. Confirmation by direct DNA sequencing of all individuals with the disorder is still challenging, and testing may not be available in local clinical practice. This disorder has a very high penetrance (development of clinical disease in a genetically affected individual). Up to $40 \%$ of affected individuals have no known family history of ADPKD.

Mutations in the PKD1 gene that are closer to the transcription start site ( $5^{\prime}$ end) potentially have a more severe effect on the translated polycystin-1 protein and are associated with earlier onset of ESRD. The PKD1 protein (polycystin-1) has multiple functions including regulation of cellular adhesion, proliferation and apoptosis. The PKD2 protein (polycystin-2) interacts with polycystin-1 and operates as a non-selective calcium channel. Polycystins are, together with the von Hippel-Lindau protein, integral components of primary renal cilia (structures on the luminal surface of renal tubular epithelial cells that act as mechanosensors and chemosensors of urine flow). Polycystic kidney disease is thus considered a ciliopathy, and its pathophysiology is best understood in this way

\section{Pathophysiology}

Abnormal primary renal cilium function in ADPKD is presumed to blunt sensing of urinary flow rates, resulting in altered renal tubular epithelial cell signalling, intracellular calcium concentrations and cyclic adenosine monophosphate (cAMP) levels (Figure 1). The cilia are connected to centrosomal structures involved in cell-cycle control. Disordered growth of renal tubular cells leads to the formation of an outpouching from the parent tubule. Less than $5 \%$ of the tubules develop an outpouching, which is the beginning of a cyst. Whereas these are initially in contact with the tubular lumen (and glomerular filtrate), they later become separate fluid-filled cystic structures lined by epithelial cells. All this abnormal cellular differentiation, maturation and apoptosis depends on the germline $P K D$ mutation acting in concert with other genetic and environmental factors, including a second somatic 'hit' on the normal PKD allele. Cyst enlargement is accompanied by gradual destruction and atrophy of the surrounding renal tissue, with ultimate progression to ESRD.

\section{Clinical course}

Although ADPKD can lead to ESRD in childhood, it more commonly occurs in middle age or later. A family history of ADPKD can be present, but the diagnosis is often made coincidentally after radiological imaging of the abdomen. Ultrasonography (Figure 2 ) is most often used to establish a diagnosis but cannot reliably exclude ADPKD in younger patients with a milder phenotype. ${ }^{4}$ Computed tomography (CT; Figures 3 and 4) and magnetic resonance imaging (MRI) have higher resolution and can be used to screen younger family members ( $<30$ years old), particularly if they are being considered as living related kidney donors. Genetic testing may be helpful particularly in families with ADPKD and a known pathogenic mutation. MRI has also been used for accurate assessment of total kidney volume in clinical trials of therapy for ADPKD. ${ }^{4}$ It is important to document kidney size as well as the location and distribution of cysts. In ADPKD, cysts tend to be distributed throughout the kidney, in comparison with some other cystic disorders, such as nephronophthisis and medullary cystic disease, in which cysts are typically located at the corticomedullary border.

ADPKD is the primary renal diagnosis in approximately $7 \%$ of UK incident ESRD patients. ${ }^{1}$ The average rate of decline in glomerular filtration rate in some series can be around 4.4-5.9 $\mathrm{ml} /$ minute per year. Risk factors for progressive renal failure include younger age at diagnosis, large kidney volumes on scan or more rapid growth over time, hypertension, male gender, gross haematuria and inheritance of a $P K D 1$ rather than $P K D 2$ mutation. For instance, inheritance of $P K D 1$ mutations confers an earlier median age of onset of ESRD (54 years) than occurs with PKD2 mutations (74 years). ${ }^{3}$ Up to $40 \%$ of patients with a confirmed diagnosis have a negative family history, reflecting either clinically silent ADPKD in a parent or a de novo mutation in a PKD gene. 
ADPKD can present at any age. The most common clinical features are urinary tract infection, loin pain, hypertension and visible haematuria. Loin pain can be secondary to rapid expansion of one or more cysts, bleeding into a cyst or urinary tract causing clot colic, cyst infection or nephrolithiasis. Cyst infection can be difficult to eradicate because larger cysts do not communicate with filtering glomeruli. The antibiotic must enter the cyst by diffusion (rather than glomerular filtration). Lipid-soluble antibiotics, such as ciprofloxacin or co-trimoxazole, are preferred as they diffuse into cyst fluid more rapidly than penicillins or aminoglycosides.

\section{Extra-renal features}

ADPKD can be considered as a systemic disorder of polycystin biology with a number of extra-renal manifestations. Hepatic cysts commonly develop later than kidney cysts, without affecting liver function, but can be complicated by cyst pain and infection (Figure 4). Massive liver cysts are more common in women than men, particularly in women who have had multiple pregnancies, suggesting a role for female sex hormones in cyst development. Rarely, liver surgery is necessary for cystic hepatomegaly that is causing pressure on surrounding anatomical structures, resulting in severe pain, poor appetite or compression of the inferior vena cava. Cysts can also be present in the spleen and pancreas.

Approximately $10 \%$ of patients with ADPKD have a strong family history of intracranial haemorrhage from a ruptured cerebral aneurysm. The risk-benefit ratio of screening asymptomatic ADPKD patients remains controversial; screening is advised only in those with a history of rupture, a family history of stroke or an occupation where sudden loss of consciousness would place the patient or others in danger. Discussion with a neurosurgeon is strongly advised before embarking upon elective scanning because of the high incidence of small aneurysms that could have limited clinical significance. For ADPKD patients presenting with acute, severe headache, rapid neurological evaluation, including CT scanning, is advisable to detect any aneurysm and blood in the subarachnoid space. Surgical clipping or endovascular occlusion of a cerebral aneurysm is recommended in patients with warning symptoms or where the aneurysm is $>7-$ $10 \mathrm{~mm}$ diameter. Other non-cystic ADPKD complications include mitral valve prolapse, aortic regurgitation, colonic diverticula and inguinal and abdominal wall herniae.

\section{Fertility and pregnancy}

Fertility in men is usually unaffected despite some having seminal or prostatic cysts. Women with normal blood pressure and renal function usually have uncomplicated pregnancies with good outcomes. Those who develop hypertension during pregnancy are more likely to have persistent hypertension after delivery. Hypertensive women are at increased risk of pre-eclampsia and fetal loss. Women who already have CKD before conception have a higher risk of hypertension, fetal loss or prematurity and progression of renal failure during pregnancy. Where possible, avoidance of oestrogen-based contraception seems sensible given the potential growth-promoting effects of these drugs on liver cysts.

\section{Treatment}

Hypertension is almost universal in ADPKD and usually develops when the kidneys have increased in size but before the onset of renal failure. Loss of normal nocturnal dipping in blood pressure in younger adults precedes the emergence of more sustained hypertension. Antihypertensive treatment reduces the risk of hypertension-related complications in CKD, but lowering blood pressure does not obviously slow the progression of ADPKD.

Hypertension can be controlled by renin-angiotensin axis blockade with angiotensin-converting enzyme (ACE) inhibitors or angiotensin receptor blockers (ARBs). Diuretics should be avoided because of experimental evidence that cyst growth is mediated by vasopressin (antidiuretic hormone). In theory, diuretic-induced depletion of intravascular volume could accelerate cyst growth by increasing vasopressin levels. In contrast, maintaining a high daily oral fluid intake suppresses vasopressin release, and this strategy could be beneficial in the long-term management of ADPKD. Dietary protein restriction has no 
significant effect on the rate of loss of renal function. Cyst drainage has been used to alleviate pain; however, there is no evidence that this intervention delays progression of renal failure and, indeed, cysts tend to recur within a matter of weeks.

Once advantage of prolonged clinical follow-up for individuals with ADPKD is advanced planning for RRT. Options for RRT included pre-emptive kidney transplant (living or deceased donor), haemodialysis or peritoneal dialysis. Peritoneal dialysis is less commonly undertaken, primarily because of the limited intraabdominal space for dialysate exchange. Interestingly, ADPKD patient survival with any form of RRT is generally better than for other non-ADPKD patients with ESRD. Nephrectomy of one or both cystic kidneys is occasionally required to accommodate a later renal transplant or for persistent pain, infection or gross haematuria.

\section{Specific therapy for ADPKD}

Research to understand the cell biology of cyst growth and animal models of polycystic kidneys has accelerated the development of potential clinical treatments for ADPKD (Figure 1). Growth of polycystic kidneys can be limited by blocking the cell membrane transporters that increase fluid secretion into cysts. ${ }^{3}$ In vivo data confirmed that vasopressin $\mathrm{V} 2$ receptor antagonists lower renal tubular epithelial cAMP levels and reduce the rate of kidney cyst growth, thereby slowing progression of CKD in animal models of polycystic kidneys.

In 2015, tolvaptan (a vasopressin antagonist) became the first UK National Institute for Health and Care Excellence (NICE)-approved treatment designed to slow the progression of cyst development and renal failure in ADPKD. Tolvaptan can be prescribed for patients with ADPKD and CKD stage 2 or 3 with rapidly declining glomerular filtration rate. The main adverse effects of tolvaptan are thirst and polyuria. In clinical trials of tolvaptan, liver function test abnormalities were more common than with placebo, so monthly monitoring of liver function for 18 months is mandatory and every 3 months thereafter. Multiple other agents including mTOR (mammalian target of rapamycin) inhibitors, somatostatin analogues, tyrosine kinase inhibitors and metformin are undergoing clinical trials. It is hoped that continued research on the biology of PKD will ultimately result in safe and cost-effective treatments that significantly extend renal survival.

Increased water intake ( $>3$ litres/day) can reduce the vasopressin-induced drive for cyst development and provides a cost-effective alternative to V2 receptor antagonism. Although this strategy currently lacks an evidence base, it seems appropriate to recommend it to patients. Finally, the use of diuretics for hypertension may be inadvisable given their tendency to cause intravascular volume depletion and increased vasopressin release (which promotes cyst development).

\section{Differential diagnosis}

Although ADPKD is the most common cystic renal disease, it is important to distinguish it from other cystic disorders (Table 2). Multiple simple renal cysts are common with advancing age, and ultrasonography criteria for ADPKD have been introduced to reduce the false-positive diagnosis rate. ${ }^{4}$ Clues to an alternative diagnosis for renal cysts include physical signs associated with tuberous sclerosis or von Hippel-Lindau disease, hearing, visual or eye problems, recurrent gout or nephrocalcinosis. Cysts can harbour or mimic neoplastic lesions. Although the cysts in ADPKD are not premalignant, they do make imaging detection of a de novo renal tumour challenging. Moreover, acquired cystic disease in patients with a long duration of ESRD is associated with an increased risk of neoplastic change. Some familial renal cancer syndromes, such as Birt-Hogg-Dubé syndrome, tuberous sclerosis and von Hippel-Lindau disease, can present with multiple cystic neoplasms.

Finally, medullary cystic disease and nephronophthisis are inherited renal disorders clinically characterized by impaired urinary concentrating ability with later progression to ESRD. Medullary cystic disease and nephronophthisis have a characteristic triad of pathological features: tubular basement 
membrane disruption, tubulo-interstitial fibrosis and cysts at the corticomedullary border. Nephronophthisis is the most frequent genetic cause of renal failure in childhood.

\section{Alport's syndrome and thin basement membrane (TBM) nephropathy Persistent asymptomatic haematuria}

Asymptomatic non-visible (microscopic) haematuria on dipstick urinalysis is a diagnostic challenge. Major causes are stratified on the basis of age. Individuals $>40$ years old are usually first investigated for renal tract neoplasms (bladder cancer, urothelial transitional cell carcinoma, renal cell carcinoma). In younger adults, non-visible haematuria is less likely to be caused by renal tract malignancy. Non-visible haematuria has a broad range of causes including glomerular pathology (e.g. immunoglobulin A (IgA) nephropathy, TBM nephropathy, Alport's syndrome); structural abnormality of the kidney (e.g. pre-symptomatic polycystic kidney disease) or metabolic disorders resulting in crystalluria (hypercalciuria, hyperuricosuria) are possible causes. Exercise (particularly long-distance running and cycling) and infection can also produce transient haematuria.

\section{Glomerular haematuria}

Glomerular haematuria can be suspected if urinary microscopy findings show dysmorphic red blood cells with or without red cell casts. Careful clinical history and examination of urine from other family members can help differentiate between IgA nephropathy, Alport's syndrome and TBM nephropathy. ${ }^{5}$ Visible haematuria associated with proteinuria in a patient without a family history of haematuria, deafness or ESRD is more likely to be IgA nephropathy. A patient with a family history of ESRD in male relatives with or without deafness is likely to have Alport's syndrome. Individuals with persistent asymptomatic non-visible haematuria that is also present in several family members without kidney failure are most likely to have TBM nephropathy (also known as benign familial haematuria). The frequency of thin glomerular basement membranes in the population could be as high as $5 \%$.

\section{Genetics}

Alport syndrome and TBM nephropathy are caused by mutations in type IV collagen genes that encode structural proteins contributing to the integrity of the glomerular basement membrane (Table 3). Alport's syndrome is rare, with an estimated prevalence of 1:50,000, and is a genetically heterogeneous disorder, with X-linked, autosomal recessive and autosomal dominant inheritance reported. ${ }^{5}$ X-linked Alport's syndrome is the most common form and is caused by mutations in the COL4A5 gene on the $\mathrm{X}$ chromosome, which encodes the a5 chain of type IV collagen (Figure 5). Father-to-son transmission cannot occur. A woman with X-linked Alport's syndrome is a heterozygous carrier: half her sons will develop Alport's syndrome and half her daughters will be carriers. Some heterozygous women, in whom one $X$ chromosome carries an Alport's syndrome mutation, exhibit haematuria, and a minority develop CKD because of skewed $\mathrm{X}$ inactivation.

The other cases of Alport's syndrome are inherited as autosomal recessive (15\%) or autosomal dominant $(20-30 \%)$ conditions with mutations arising in the COL4A3 or COL4A4 genes on chromosome 2 , which encode the $\alpha 3$ and $\alpha 4$ chains, respectively, of type IV collagen. TBM nephropathy is transmitted as an autosomal dominant disorder with a heterozygous defect in either the COL4A3 or COL4A4 gene (Figure $5)$.

\section{Pathogenesis}

The $\alpha 3, \alpha 4$ and $\alpha 5$ chains of type IV collagen are expressed in the basement membranes of the glomerulus, eye and cochlea. Mutations in the genes encoding these chains can cause disrupted assembly of type IV collagen, leading to failure of basement membrane properties, and, in the glomerulus, increased susceptibility to basement membrane splitting and eventual glomerulosclerosis, which is typically seen in Alport's syndrome. Anterior lenticonus, resulting from thinning of the lens capsule (basement membrane), 
is a pathognomonic sign in the eye of $20-30 \%$ of male patients with X-linked Alport syndrome. Hearing loss is caused by impaired adhesion of the organ of Corti to the basilar membrane in the inner ear.

TBM nephropathy is associated with collagen defects causing diffuse thinning of the glomerular basement membrane that is severe enough to result in persistent haematuria. However, in most cases, the pathology does not progress to glomerular sclerosis, and the proteinuria and declining renal function typical of Alport's syndrome do not usually occur.

\section{Clinical features}

Persistent non-visible haematuria is usual in boys with Alport's syndrome. Progressive CKD is associated with hypertension and proteinuria. ESRD typically occurs before the fourth decade. The rate of loss of kidney function partly correlates with the underlying gene mutation, with gene deletions resulting in more severe disease than missense mutations. High-tone sensorineural hearing loss and eye changes also progress. Rarely, oesophageal leiomyomatosis associated with COL4A6 mutations is seen in conjunction with Alport's syndrome and can cause severe dysphagia.

A diagnosis of Alport's syndrome can be established by skin biopsy and immunohistochemical staining with an antibody against the a5 chain of type IV collagen. ${ }^{5}$ Absence of a 5 chain staining in a male patient or a mosaic pattern in a female confirms the diagnosis of X-linked Alport's syndrome or female carrier status. Patients with autosomal dominant and autosomal recessive Alport's syndrome have normal skin immunohistochemistry. If skin biopsy is not helpful, the diagnosis can be made on renal biopsy with immunohistochemical staining for components of type IV collagen. Molecular genetic testing using nextgeneration sequencing is becoming the standard diagnostic test. DNA sequencing is non-invasive and accurate, and can simultaneously examine the relevant collagen genes to identify specific mutations.

Alport's syndrome has no specific treatment and care is largely supportive, including control of hypertension and preparation for RRT. Registry data support the use of ACE inhibitor or ARB therapy in delaying time to ESRD. Individuals with Alport's syndrome should avoid ototoxic medication such as aminoglycosides and ensure they minimize industrial noise exposure where relevant. Pre-emptive live donor kidney transplant is the ideal form of RRT. Alport's syndrome obviously does not recur in renal transplants, but a small minority of transplant recipients develop antiglomerular basement membrane antibody disease. ${ }^{5}$ This results from the recipient's immune system encountering normal type IV collagen for the first time and mounting an antibody response to the 'foreign' antigen.

No intervention is required for non-visible haematuria in people with TBM nephropathy provided they have normal blood pressure, absence of proteinuria and normal renal function. Pregnancy complications, including pre-eclampsia, are no more common in those with TBM nephropathy. Even though the condition typically follows a benign course, it is prudent to recommend measurement of blood pressure, proteinuria and renal function 1-2-yearly by the patient's general practitioner. 


\begin{tabular}{|c|c|c|c|}
\hline \multicolumn{4}{|l|}{ Renal tubular disorders } \\
\hline Disease & Phenotype & Affected protein & Comments \\
\hline Liddle's syndrome (AD) & $\begin{array}{l}\text { Hypertension, hypokalaemia, } \\
\text { hypoaldosteronism }\end{array}$ & $\begin{array}{l}\beta \text { unit of epithelial sodium } \\
\text { channel }\end{array}$ & $\begin{array}{l}\text { Treat with low-sodium diet and } \\
\text { potassium-sparing diuretics (e.g. } \\
\text { amiloride) }\end{array}$ \\
\hline \multirow[t]{2}{*}{ Bartter's syndrome (AR) } & Hypokalaemia, hypercalciuria & $\begin{array}{l}\mathrm{Na}^{+}-\mathrm{K}^{+}-\mathrm{Cl}^{-} \text {co-transporter } \\
\mathrm{K}^{+} \text {channel }\end{array}$ & \multirow[t]{2}{*}{$\begin{array}{l}\text { Usually manifests in infancy. Phenotype } \\
\text { identical to those taking loop diuretics }\end{array}$} \\
\hline & & $\mathrm{Cl}^{-}$channel & \\
\hline Gitelman's syndrome (AR) & $\begin{array}{l}\begin{array}{l}\text { Hypokalaemia, } \\
\text { hypomagnesaemia, hypocalciuria }\end{array} \\
\end{array}$ & $\mathrm{Na}^{+}-\mathrm{Cl}^{-}$co-transporter & $\begin{array}{l}\text { Phenotype identical to those taking } \\
\text { thiazide diuretics }\end{array}$ \\
\hline \multirow[t]{2}{*}{$\begin{array}{l}\text { Nephrogenic diabetes insipidus } \\
\text { (XR, AR and AD forms) }\end{array}$} & \multirow[t]{2}{*}{ Polyuria } & $\begin{array}{l}\text { Arginine vasopressin } \\
\text { receptor } 2\end{array}$ & \multirow[t]{2}{*}{$\begin{array}{l}\text { Lithium and hypercalcaemia should be } \\
\text { excluded as acquired causes }\end{array}$} \\
\hline & & Aquaporin 2 & \\
\hline \begin{tabular}{|l|} 
Dent's disease (X-linked \\
hypercalciuric nephrolithiasis)
\end{tabular} & $\begin{array}{l}\text { Renal calculi, hypercalciuria, } \\
\text { nephrocalcinosis, proteinuria }\end{array}$ & Clc-5 (a chloride channel) & \\
\hline
\end{tabular}

Table 1 


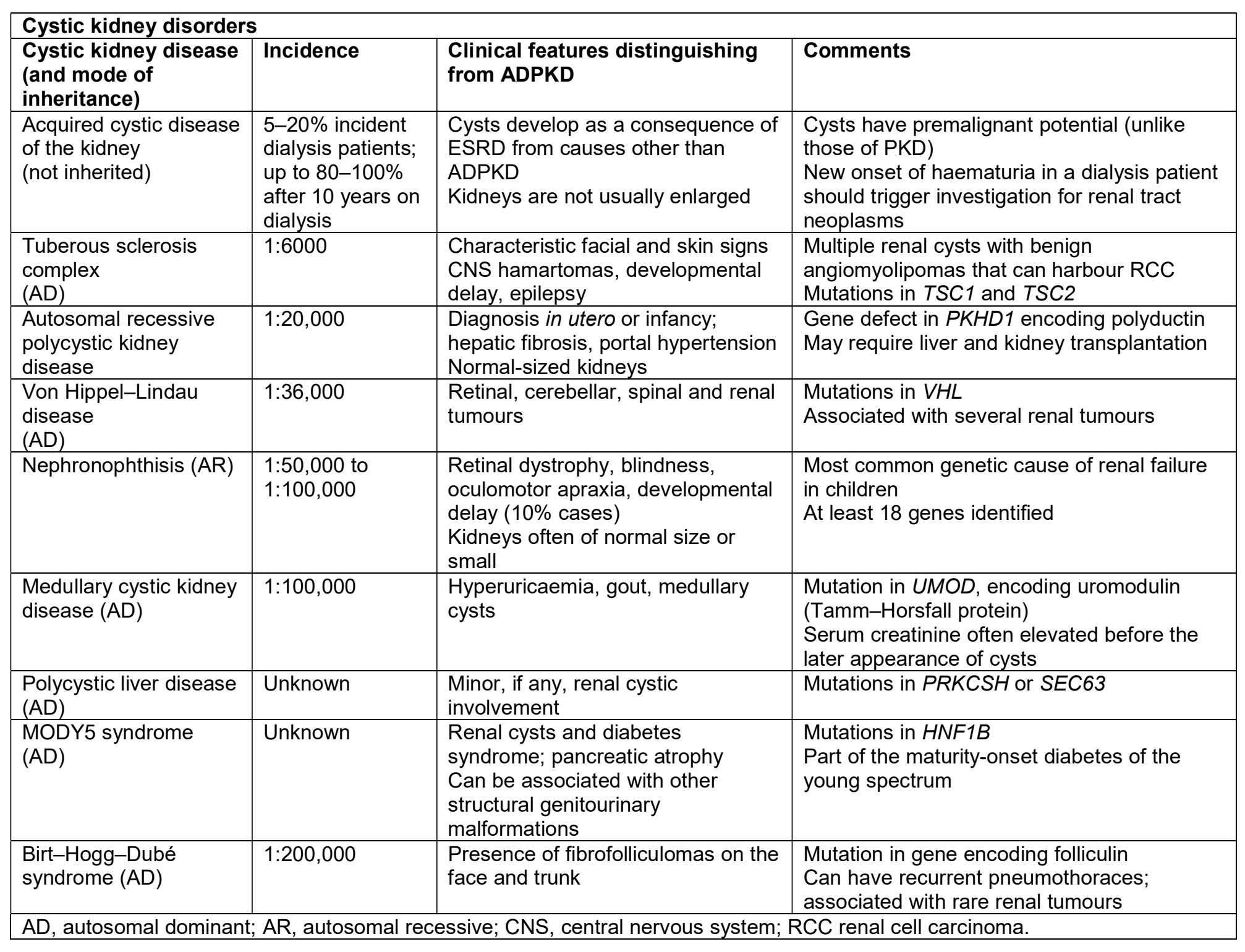


Table 2 


\begin{tabular}{|c|c|c|c|c|}
\hline \multicolumn{5}{|c|}{ Hereditary glomerular disorders mimicking Alport's syndrome } \\
\hline \multirow{3}{*}{$\begin{array}{l}\text { Alport's syndrome } \\
\text { (prevalence estimate 1:50,000) }\end{array}$} & X-linked & $\mathrm{Xq}$ & COL4A5 & a5 chain of collagen IV \\
\hline & AR & $\begin{array}{l}2 q 35- \\
q 37\end{array}$ & COL4A3/COL4A4 & a3/a4 chain \\
\hline & $A D$ & $\begin{array}{l}2 q 35- \\
q 37\end{array}$ & COL4A3/COL4A4 & $\alpha 3$ or $\alpha 4$ chain of collagen IV \\
\hline $\begin{array}{l}\text { TBM nephropathy } \\
\text { (prevalence estimate } 1 \% \text { of population) }\end{array}$ & $A D$ & $2 q$ & COL4A3/COL4A4 & $\alpha 3$ or $\alpha 4$ chain of collagen IV \\
\hline \begin{tabular}{|l|} 
MYH9-related diseases: Epstein's and \\
Fechtner's syndromes (sensorineural deafness, \\
congenital thrombocytopenia with giant \\
platelets, nephropathy) \\
(prevalence estimate $1: 200,000)$
\end{tabular} & AD & $22 q$ & MYH9 & MYH9 \\
\hline
\end{tabular}

Table 3 


\section{FIGURE CAPTIONS}

\section{Figure 1}

Intracellular signalling disruption in ADPKD

Schematic of a renal tubular epithelial cell and its primary cilium. Mutations the PKD1 and PKD2 lead to relative or absolute loss of function of the polycystin complex within the primary cilium. Reduced intracellular calcium influx occurs, and the compensatory increase in cAMP levels promotes fluid secretion and cell proliferation. Cell proliferation is driven in part by up-regulation of the mTOR pathway secondary to defective ciliary function. Vasopressin (antidiuretic hormone) acting through V2 receptors increases cAMP levels, while somatostatin inhibits cAMP generation. These signalling pathways are the targets of the therapeutic agents sirolimus, tolvaptan and octreotide.

PC1, polycystin-1; PC2, polycystin-2.

\section{Figure 2}

Assessment of ADPKD by ultrasonography, demonstrating multiple fluid-filled kidney cysts.

\section{Figure 3}

Assessment of ADPKD by CT demonstrating massive cystic enlargement of both kidneys, which occupy most of the abdominal cavity

\section{Figure 4}

Assessment of ADPKD by CT demonstrating liver and kidney cysts.

\section{Figure 5}

Inheritance of Alport's syndrome and TBM nephropathy. 


\section{KEY REFERENCES}

1 Hole B, Gilg J, Casula A, Methven S, Casledine C. UK Renal Registry 20th Annual Report: Chapter 1 UK renal replacement therapy adult incidence in 2016: national and centre-specific analyses.

https://www.renalreg.org/wp-content/uploads/2018/06/01-Chap01-1.pdf (accessed 13 Jan 2019).

2 Leung JC. Inherited renal diseases. Curr Pediatr Rev 2014; 10: 95-100.

3 Bergmann C, Guay-Woodford LM, Harris PC, et al. Polycystic kidney disease. Nat Rev Dis Primers 2018; 4: 50.

4 Pei Y, Hwang YH, Conklin J, et al. Imaging-based diagnosis of autosomal dominant polycystic kidney disease. J Am Soc Nephrol 2015; 26: 746-53.

5 Kashtan C. Alport syndrome: facts and opinions. F1000Res 2017; 6: 50.

\section{TEST YOURSELF}

To test your knowledge based on the article you have just read, please complete the questions below. The answers can be found at the end of the issue or online here.

\section{Question 1}

An 18-year-old man presented with persistent non-visible haematuria initially identified during an armed forces medical examination. He was fit and training for a marathon. There was a family history of endstage renal disease. An uncle had been given a renal transplant at age 29 years.

On clinical examination, his blood pressure was $115 / 78 \mathrm{mmHg}$. Urinalysis showed $3+$ blood and 2+ protein.

Investigations $U$ rine albumin:creatinine ratio $93 \mathrm{mg} / \mathrm{mmol}(<3)$

-Serum creatinine 153 micromol/litre (70-110)

-Estimated glomerular filtration rate $54.9 \mathrm{ml} / \mathrm{min} / 1.73 \mathrm{~m}^{2}(>60)$

What is the most likely cause for the persistent haematuria?
A. Alport's syndrome
B. Hypercalciuria
C. Immunoglobulin A nephropathy
D. Polycystic kidney disease
E. Thin basement membrane nephropathy

Correct answer: A. All answers are possible but Alport's syndrome is most likely as the patient has renal impairment and there is a family history of early onset end-stage renal disease in a male relative. Polycystic kidney disease $(D)$ is possible but the earlier age of onset of chronic kidney disease in the patient (and endstage renal disease in the uncle) would be atypical. Immunoglobulin A nephropathy (C) is possible but typically would be associated with hypertension. Thin basement membrane nephropathy $(E)$ an hypercalciuria $(E)$ could both cause haematuria in a young man but are less likely to cause renal impairment in early adulthood.

\section{Question 2}

A 48-year-old woman attended for further discussion of options for renal replacement therapy. She had polycystic kidney disease. Her kidney function, as measured by estimated glomerular filtration rate, was 
decreasing by $4 \mathrm{ml} /$ minute per year. She had well-controlled hypertension but no other co-morbidities.

She was a non-smoker. Her body mass index was $26 \mathrm{~kg} / \mathrm{m}^{2}$.

\section{Investigations}

-Serum creatinine 273 micromol/litre (70-110)

-Estimated glomerular filtration rate $17.1 \mathrm{ml} / \mathrm{min} / 1.73 \mathrm{~m}^{2}(>60)$

Which renal replacement therapy option will provide the best long-term survival for this woman?
A. Ambulatory peritoneal dialysis
B. Deceased donor kidney transplant
C. Home haemodialysis
D. Hospital-based haemodialysis
E. Live donor kidney transplant

Correct answer: E. All answers are potential renal replacement therapy options. Live donor kidney transplant $(E)$ is associated with optimal long-term survival. If a live donor was not available then deceased donor kidney transplant (B) would be next best option for survival. If a transplant procedure could not be offered then home haemodialysis $(\mathrm{C})$ is preferred as survival on home haemodialysis can almost match deceased donor transplant. Hospital-based haemodialysis (D) offers better long term survival than peritoneal dialysis $(A)$ mainly due to peritoneal dialysis technique failure in the first 5 years of use.

\section{Question 3}

A 37-year-old man presented with kidney function, as measured by estimated glomerular filtration rate, decreasing by $4 \mathrm{ml} /$ minute per year. He had polycystic kidney disease and hypertension treated with ramipril and amlodipine.

On clinical examination, his heart rate was 76 beats/minute, and blood pressure 139/83 $\mathrm{mmHg}$. Both kidneys were palpable. There was no oedema.

\section{Investigations}

-Serum creatinine143 micromol/litre (70-110)

-Estimated glomerular filtration rate $51.3 \mathrm{ml} / \mathrm{min} / 1.73 \mathrm{~m}^{2}(>60)$

-Serum bicarbonate $22 \mathrm{mmol} / \mathrm{itre}(24-29)$

What additional therapy might slow the progression of his chronic kidney disease?
A. Bisoprolol
B. Octreotide
C. Sirolimus
D. Sodium bicarbonate
E. Tolvaptan

Correct answer: E. Tolvaptan is licensed for progressive renal failure in stage 2 and stage 3 chronic kidney disease (CKD) associated with autosomal dominant progressive kidney disease (ADPKD). Octreotide (B) and sirolimus (C) have been used in clinical trials of ADPKD, but without proven efficacy. Sodium bicarbonate (D) may delay progression of CKD but can cause worsening of hypertension (because of the sodium load). Bisoprolol (D) can be used for hypertension but is unlikely to affect progression of the patient's CKD. 